\title{
Position-dependent noncommutative products: Classical construction and field theory
}

\author{
V. Gayral ${ }^{\mathrm{a}, 1}$, J.M. Gracia-Bondía ${ }^{\mathrm{b}}$, F. Ruiz Ruiz ${ }^{\mathrm{b}}$ \\ a Centre de Physique Théorique, UMR 6207, 13288 Marseille, France \\ ${ }^{\mathrm{b}}$ Departamento de Física Teórica I, Universidad Complutense de Madrid, 28040 Madrid, Spain
}

Received 8 April 2005; accepted 8 August 2005

Available online 31 August 2005

\begin{abstract}
We look in Euclidean $\mathbb{R}^{4}$ for associative star products realizing the commutation relation $\left[x^{\mu}, x^{\nu}\right]=$ $i \Theta^{\mu \nu}(x)$, where the noncommutativity parameters $\Theta^{\mu \nu}$ depend on the position coordinates $x$. We do this by adopting Rieffel's deformation theory (originally formulated for constant $\Theta$ and which includes the Moyal product as a particular case) and find that, for a topology $\mathbb{R}^{2} \times \mathbb{R}^{2}$, there is only one class of such products which are associative. It corresponds to a noncommutativity matrix whose canonical form has components $\Theta^{12}=-\Theta^{21}=0$ and $\Theta^{34}=-\Theta^{43}=\theta\left(x^{1}, x^{2}\right)$, with $\theta\left(x^{1}, x^{2}\right)$ an arbitrary positive smooth bounded function. In Minkowski space-time, this describes a position-dependent space-like or magnetic noncommutativity. We show how to generalize our construction to $n \geqslant 3$ arbitrary dimensions and use it to find traveling noncommutative lumps generalizing noncommutative solitons discussed in the literature. Next we consider Euclidean $\lambda \phi^{4}$ field theory on such a noncommutative background. Using a zeta-like regulator, the covariant perturbation method and working in configuration space, we explicitly compute the UV singularities. We find that, while the two-point UV divergences are nonlocal, the four-point UV divergences are local, in accordance with recent results for constant $\Theta$.

(C) 2005 Elsevier B.V. All rights reserved.
\end{abstract}

PACS: 11.10.-z; 11.10.Lm; 11.10.Gh

Keywords: Noncommutative field theory; Nonlocal models; Renormalization; Generalized deformations

E-mail addresses: gayral@cpt.univ-mrs.fr (V. Gayral), ferruiz@ fis.ucm.es (F. Ruiz Ruiz).

1 Also at Université de Provence, France. 


\section{Introduction}

Seiberg and Witten have argued [1] that the endpoints of open strings in a magnetic field background live on a Moyal (hyper)plane. The mathematical properties of these spaces were familiar to physicists since the fifties, especially in connection with the phase-space formulation of quantum mechanics [2]. To this knowledge must be added the recent proof [3] that Moyal planes are spectral triples, that is, virtual spin manifolds in the sense of Connes [4,5]. Yet, despite all these good properties, quantum field theory on Moyal planes (NCFT for short) is a difficult construct.

The problems of NCFT arise with renormalization and are ultimately caused by the nonlocal nature of the Moyal 'star' product. One of these problems is the conciliation of unitarity with the locality of the counterterms needed to subtract UV divergences. Indeed, whereas on the one hand it has been proved [6] that noncommutativity matrices $\Theta=\left[\Theta^{\mu \nu}\right]$ with only space-space components yield one-loop renormalized Green functions consistent with unitarity, on the other hand it has been shown that such Green functions do not define an effective action [7] (see Ref. [8] for an argument in terms of Wick products). This indicates that noncommutativity matrices with nonvanishing space-time components should be investigated. Some progress along this line is taking place $[9,10]$. Another source of trouble is the occurrence of tachyonic instabilities [11, 12] already at first order in perturbation theory. Such instabilities, and partly the nonlocality of the UV counterterms for space-space matrices $\Theta$, are linked to the by now well-known UV/IR mixing phenomenon [13]. Fortunately enough, there is a cure for this problem, since such instabilities can be eliminated and the UV/IR mixing avoided by introducing supersymmetry [12,14]. So, all in all, progress is being made little by little. In this respect it is worth mentioning the general belief that by removing the UV/IR mixing the renormalization program is feasible at higher orders in perturbation theory.

In this paper we take a different view and investigate almost unexplored territory: NCFT on nonconstant (i.e. coordinate-dependent) noncommutativity spaces. We have several motivations for this enterprise. The first one comes from the appearance of nonconstant noncommutativity in various contexts, in particular from the continued development of string theory; a literature sample may include Refs. [15-20]. The second one is that, partly motivated by the former and by the hope of dealing with gravity, it is natural to look at the noncommutativity parameters as dynamical variables. A step in this direction is to make them dependent on coordinates. One could count, in the third place, the hope of finding a transitional regime between the noncommutative and the commutative realms. This hope seems to be misplaced, at least for nonsupersymmetric theories, as the UV/IR phenomenon and the generation of IR singularities when the noncommutativity parameters approach zero are not overcome. Last, but not least, the problem has interest from the viewpoint of the general theory of noncommutative spaces, for there are not so many examples of these.

As we will see below, there are ways to realize the commutator

$$
\left[x^{\mu}, x^{\nu}\right]=i \Theta^{\mu \nu}(x),
$$

where the matrix $\Theta(x)=\left[\Theta^{\mu \nu}(x)\right]$ depends on coordinates, by means of star products. Nonassociative star products have been explicitly used in [21]. However, for field theory and also for the sake of defining noncommutative spaces with a minimum set of bona fide properties, one wants an associative product with more or less the same basic properties as Moyal's. So we must first investigate the question of whether there are admissible associative generalizations of the Moyal 
product with position-dependent noncommutativity. We will do this by using Rieffel's deformation procedure [22], a true and tested recipe for generating suitable noncommutative spaces with constant noncommutativity, and studying under what conditions it can be extended to cover nonconstant noncommutativity. This by itself is not a trivial issue. In fact, in the literature one often finds Eq. (1.1), but no direct explicit realizations are given. As already declared, we want to do quantum field theory on $\mathbb{R}^{4}$ with nonconstant $\Theta(x)$, so we will be interested in nonperiodic star products of functions (fields) defined on $\mathbb{R}^{4}$. We will consider Euclidean $\mathbb{R}^{4}$ with topology $\mathbb{R}^{2} \times \mathbb{R}^{2}$ and use coordinates $x=\left(\bar{x}^{1}, \bar{x}^{2}, \tilde{x}^{1}, \tilde{x}^{2}\right)$ to exploit the canonical form of antisymmetric matrices $\Theta(x)$ in four dimensions and the fact that $\Theta(x)$ can only have rank 4, 2 or 0 . The outcome of this investigation is surprising because of the uniqueness of its result. Namely, the only noncommutativity matrix $\Theta(x)$ yielding an associative nonconstant star product of the desired type will turn out to have rank 2 and entries $\Theta^{\bar{i} \bar{j}}=0$ and $\Theta^{\tilde{a} \tilde{b}}=\varepsilon^{\tilde{a} \tilde{b}} \theta(\bar{x})$, with $\theta(\bar{x})$ an arbitrary positive and suitably bounded function, or the other way around. This corresponds in the Minkowskian framework to an $x$-dependent generalization of space-like or magnetic constant noncommutativity. We will see all this in Section 2. We also give there an example of a nonconstant star product for $\mathbb{R}^{4}$ foliated by 3 -spheres.

The construction in Section 2 of a position-dependent star product makes an obvious exercise to generalize the noncommutative soliton solutions in the literature [23] to traveling noncommutative lumps. This is done in Section 3.

Once we have a sensible nonconstant star product, we move on to consider quantum $\lambda \phi^{4}$ field theory for such a product. We will define the quantum theory through the effective action and study whether the theory is renormalizable at one loop. Because of the presence of the generic function $\theta(\bar{x})$ we are led to work in position space. To compute one-loop radiative corrections and examine their UV singularities we will use the covariant perturbation method [24], due to Barvinsky and Vilkovisky, proposed as an alternative to the Schwinger-DeWitt technique [25] and tested in the quantization of fields coupled to gravitational backgrounds. We discuss in Section 4 the particulars of the method when applied to our problem.

Sections 5 and 6 contain the analysis of the two and four-point parts of the effective action. Despite the nonlocalities of the theory, we succeed in completely characterizing the UV divergences in the model. While the four-point divergences are completely local and can be subtracted by local counterterms, the two-point divergences are nonlocal and cannot be removed by local counterterms. This poses an obstacle to traditional perturbative renormalization and shows that the $\lambda \phi^{4}$ model can only be viewed at this stage as an effective theory. This is in complete accordance with the $\theta$-constant case. Section 7 contains our conclusion. We include at the end two mathematical Appendices A and B-which the not mathematically inclined reader may omit- to put our discussion on a rigorous footing.

We want to emphasize at the outset that we are not claiming ours is the only method to construct associative star products compatible with position-dependent commutation relations. Other methods are found in the literature, for instance in relation with Kontsevich's formality for Poisson structures [26], or as deformations of the commutation relation (1.1) for constant $\Theta$ [27]. Those methods have in common that the noncommutativity matrix is regarded as a perturbation, and the expansions in $\Theta$ are analytically uncontrolled. For the noncommutative products obtained in Ref. [15] as symplectic reductions of Moyal algebras to function groups, this is also true in practice, although not in principle. Our approach, however, is not perturbative in $\Theta$. As we intend here to do field theory, well-definiteness of the various objects involved in our analysis becomes crucial, so our preferences go to deformations with good analytical properties, and in that respect Rieffel's approach singles itself. 


\section{Construction of star products with nonconstant $\Theta(x)$}

We start by recalling the construction of the original Moyal product. For any finite dimension $k \geqslant 2$, we denote by $\Theta$ a real nondegenerate skew-symmetric $k \times k$ matrix. The nondegeneracy condition implies even dimension, so that $k=2 N$ with $N \geqslant 1$. Given now two suitable functions $f$ and $g$ on $\mathbb{R}^{2 N}$, their Moyal product is a function of the same type defined as

$$
\left(f \star_{\Theta} g\right)(x)=\frac{1}{\pi^{2 N} \operatorname{det} \Theta} \int d^{2 N} y d^{2 N} z f(x+y) g(x+z) e^{-2 i y \Theta^{-1} z} .
$$

Since $y$ and $z$ are points in space-time, the entries of $\Theta$ have the dimensions of an area. The Moyal product clearly satisfies the trace property, for

$$
\int d^{2 N} x\left(f \star_{\Theta} g\right)(x)=\int d^{2 N} x f(x) g(x) .
$$

This property allows to extend definition (2.1) to (huge) appropriately chosen function and distribution multiplier spaces [30]. In particular, the Moyal product of periodic functions is periodic, and thus noncommutative tori are subsumed in Moyal theory.

The crucial remark by Rieffel is that Eq. (2.1) may be rewritten as

$$
\left(f \star_{\Theta} g\right)(x)=\frac{1}{(2 \pi)^{k}} \int d^{k} y d^{k} z f\left(x-\frac{1}{2} \Theta y\right) g(x+z) e^{-i y z} .
$$

This formula is the starting point for a far-reaching deformation theory of algebras, in which the $\Theta$-parameters carry actions of continuous Abelian groups $\mathbb{R}^{m} \times \mathbb{T}^{l-m}$ and no longer nondegeneracy/even dimension are issues. For instance, a generalization of Eq. (2.2) in terms of such actions, by isometries on suitable Riemannian manifolds, has been analyzed in Refs. [31,32], with most properties of the Moyal product being kept.

To begin our study, we recall the well-known fact that any antisymmetric constant matrix $\Theta$ can be written in the form

$$
\Theta=A^{t}\left(\begin{array}{cc}
\zeta S & 0 \\
0 & \theta S
\end{array}\right) A \quad \text { with } S=\left(\begin{array}{cc}
0 & 1 \\
-1 & 0
\end{array}\right),
$$

where $A$ is orthogonal, $A^{t}=A^{-1}$, and $\zeta$ and $\theta$ are constants. Note that, being $A$ orthogonal, the previous transformation is both a congruence and a similarity. Let us assume now that two antisymmetric matrices $\Theta$ and $\Theta^{\prime}$ are related by such a transformation,

$$
\Theta^{\prime}=A^{t} \Theta A=: \mathcal{A}[\Theta],
$$

and let us define local transformations

$$
A f(x)=f\left(A^{-1} x\right) .
$$

As is well known, it then follows that the corresponding star products $\star_{\Theta}$ and $\star_{\Theta^{\prime}}$ are covariant under $A$, meaning that there exists an algebra isomorphism such that, with a slight abuse of notation,

$$
A(f \star \mathcal{A}[\Theta] g)(x)=A f(x) \star_{\Theta} A g(x) .
$$

In particular, if $\Theta$ and $A$ commute, there is equivariance, i.e.

$$
A\left(f \star_{\Theta} g\right)(x)=A f(x) \star_{\Theta} A g(x) .
$$


For instance, if

$$
\Theta=\left(\begin{array}{cc}
\zeta S & 0 \\
0 & \theta S
\end{array}\right)
$$

the star product is equivariant under transformations of the group $S O(2 ; \mathbb{R}) \times S O(2 ; \mathbb{R})$ —actually under the bigger group $S L(2 ; \mathbb{R}) \times S L(2 ; \mathbb{R})$ of symplectic transformations.

For $x$-dependent $\Theta$ we adopt Rieffel's formula (2.2) as our starting point. In this case it is natural to consider a large 'gauge' group $\mathcal{F}\left(\mathbb{R}^{4}, O(4 ; \mathbb{R})\right.$ ) of transformations

$$
A(x) f(x)=f\left(A^{-1}(x) x\right)
$$

for suitably well-behaved orthogonal-matrix valued functions $A(x)$. Then it is not difficult to see that, instead of Eq. (2.3), with $A^{t}(x) \Theta(y) A(x)=: \mathcal{A}(x)[\Theta(y)]$, the covariance property reads:

$$
A(x)(f \star \mathcal{A}(x)[\Theta(A(x) x)] g)(x)=A(x) f(x) \star_{\Theta(x)} A(x) g(x) .
$$

Thus the product $\star \mathcal{A}(x)[\Theta(A(x) x)]$ is associative if and only if $\star \Theta(x)$ is. This observation allows us to reduce the study of associativity of $\star \Theta(x)$ to the case in which the matrix $\Theta(x)$ is brought into the canonical form

$$
\Theta(x)=\left(\begin{array}{cc}
\zeta(x) S & 0 \\
0 & \theta(x) S
\end{array}\right) \quad \text { with } S=\left(\begin{array}{cc}
0 & 1 \\
-1 & 0
\end{array}\right),
$$

$\zeta(x)$ and $\theta(x)$ being functions of $x$. Henceforth we suppose that this reduction has taken place. Recalling that for a point $x \in \mathbb{R}^{4}$ we are writing $x=(\bar{x}, \tilde{x})=\left(\bar{x}^{i}, \tilde{x}^{a}\right)$, with $i, a=1,2$, the latter defines the star product of two functions $f$ and $g$ on $\mathbb{R}^{4}$ as

$$
\begin{aligned}
\left(f \star_{\Theta} g\right)(x)= & \frac{1}{(2 \pi)^{4}} \int d^{4} y d^{4} z f\left(\bar{x}-\frac{1}{2} \zeta(x) S \bar{y}, \tilde{x}-\frac{1}{2} \theta(x) S \tilde{y}\right) g(\bar{x}+\bar{z}, \tilde{x}+\tilde{z}) \\
& \times e^{-i(\bar{y} \bar{z}+\tilde{y} \tilde{z})} .
\end{aligned}
$$

For this definition to be pertinent, we need two conditions. First, it must realize the commutation relations

$$
\left[\bar{x}^{i}, \bar{x}^{j}\right]=i \Theta^{i j}(x)=i \varepsilon^{i j} \zeta(x), \quad\left[\tilde{x}^{a}, \tilde{x}^{b}\right]=i \Theta^{a b}(x)=i \varepsilon^{a b} \theta(x), \quad\left[\bar{x}^{i}, \tilde{x}^{a}\right]=0,
$$

where $\varepsilon^{12}=1$. Secondly, it must satisfy the basic properties of the ordinary $\Theta$-constant Moyal product, associativity among them. In this regard it is worth emphasizing that the entanglement caused by a nonconstant $\Theta$ in (2.4) threatens associativity.

In this section we study whether the commutation relations are realized and find the constraints that associativity imposes on the functions $\zeta(x)$ and $\theta(x)$. Clearly the matrix $\Theta(x)$ will have rank 4, 2 or 0 . For analytical reasons discussed later, we take the functions $\zeta(x)$ and $\theta(x)$ either zero or positive everywhere. This implies that we restrict ourselves to constant rank. Rank 4 corresponds to both functions $\zeta(x)$ and $\theta(x)$ being different from zero for all $x$, rank 2 corresponds to the product $\zeta(x) \theta(x)$ vanishing for all $x$ and rank 0 to both being zero. We do not consider the rank 0 case since it corresponds to the ordinary commutative product. Let us consider the rank 4 and rank 2 cases separately. 
2.1. Case 1: $\operatorname{rank}(\Theta)=4$

Using Eq. (2.4) for the product $\bar{x}^{i} \star_{\Theta} \bar{x}^{j}$ we have

$$
\bar{x}^{i} \star_{\Theta} \bar{x}^{j}=\frac{1}{(2 \pi)^{4}} \int d^{2} \bar{y} d^{2} \tilde{y} d^{2} \bar{z} d^{2} \tilde{z}\left[\bar{x}^{i}-\frac{1}{2} \zeta(x) \varepsilon^{i k} \bar{y}^{k}\right]\left[\bar{x}^{j}+\bar{z}^{j}\right] e^{-i(\bar{y} \bar{z}+\tilde{y} \tilde{z})} .
$$

Expanding the product in the integrand and integrating over $\tilde{y}$ and $\tilde{z}$ we have

$$
\bar{x}^{i} \star_{\Theta} \bar{x}^{j}=\frac{1}{(2 \pi)^{2}} \int d^{2} \bar{y} d^{2} \bar{z}\left[\bar{x}^{i} \bar{x}^{j}+\bar{x}^{i} \bar{z}^{j}-\frac{1}{2} \zeta(x) \varepsilon^{i k} \bar{y}^{k} \bar{x}^{j}-\frac{i}{2} \zeta(x) \varepsilon^{i k} \bar{y}^{k} \frac{\partial}{\partial \bar{y} j}\right] e^{-i \bar{y} \bar{z}} .
$$

Integrating now over $\bar{y}$ and $\bar{z}$ we obtain the ordinary product $\bar{x}^{i} \bar{x}^{j}$ for the first term, zero for the second and third terms and $\frac{1}{2} \zeta(x) \varepsilon^{i j}$ for the fourth term. Hence

$$
\bar{x}^{i} \star_{\Theta} \bar{x}^{j}=\bar{x}^{i} \bar{x}^{j}+\frac{i}{2} \zeta(x) \varepsilon^{i j} .
$$

It then follows that the generalized Rieffel formula (2.4) realizes the first commutation relation in Eq. (2.5). Analogous arguments show that the other two commutation relations in (2.5) are also implemented by definition (2.4).

We next discuss associativity,

$$
\left(f \star_{\Theta} g\right) \star_{\Theta} h=f \star_{\Theta}\left(g \star_{\Theta} h\right) .
$$

As is well known, a necessary condition, though not sufficient, for associativity is the Jacobi identity

$$
\left[[f, g]_{\star_{\Theta}}, h\right]_{\star_{\Theta}}+\left[[h, f]_{\star_{\Theta}}, g\right]_{\star_{\Theta}}+\left[[g, h]_{\star_{\Theta}}, f\right]_{\star_{\Theta}}=0,
$$

where we have written $[f, g]_{\star_{\Theta}}=f \star_{\Theta} g-g \star_{\Theta} f$. Let us study whether it is fulfilled, prior to moving on to associativity. For $f=\bar{x}^{i}, g=\bar{x}^{j}$ and $h=\bar{x}^{k}$, since the indices $i, j, k$ may only take values in the range 1,2 , two of them must be equal, say $i=j$. Then the identity (2.7) becomes

$$
\left[\left[\bar{x}^{k}, \bar{x}^{i}\right]_{\star \Theta}, \bar{x}^{i}\right]_{\star \Theta}+\left[\left[\bar{x}^{i}, \bar{x}^{k}\right]_{\star \Theta}, \bar{x}^{i}\right]_{\star \Theta}=0,
$$

which is trivially satisfied. The same argument shows that the Jacobi identity also holds for $f=\tilde{x}^{a}, g=\tilde{x}^{b}$ and $h=\tilde{x}^{c}$. Now, for $f=\bar{x}^{i}, g=\bar{x}^{j}$ and $h=\tilde{x}^{a}$, the commutation relations (2.5) give for Eq. (2.7)

$$
\left[\zeta(x), \tilde{x}^{a}\right]_{\star \Theta}=0 .
$$

As we are assuming $\theta(x) \neq 0$, this requires the function $\zeta(x)$ not to depend on $\tilde{x}$. Analogous arguments show that the Jacobi identity for $f=\tilde{x}^{a}, g=\tilde{x}^{b}$ and $h=\tilde{x}^{i}$ demands the function $\theta(x)$ not to depend on $\bar{x}$. We then have $\zeta(x)=\zeta(\bar{x})$ and $\theta(x)=\theta(\tilde{x})$. In other, words the noncommutativity matrix is the direct sum of two $2 \times 2$ matrices that do not speak to each other.

Let us now consider associativity itself. Using (2.4) and similar arguments as for the study of the commutation relations, it is easy to see that

$$
\left(\bar{x}^{i} \star_{\Theta} \bar{x}^{j}\right) \star_{\Theta} \bar{x}^{k}-\bar{x}^{i} \star_{\Theta}\left(\bar{x}^{j} \star_{\Theta} \bar{x}^{k}\right)=-\frac{1}{4} \zeta(\bar{x})\left[\varepsilon^{i j} \varepsilon^{k r} \frac{\partial \zeta}{\partial \bar{x}^{r}}+\varepsilon^{j k} \varepsilon^{i r} \frac{\partial \zeta}{\partial \bar{x}^{r}}\right] .
$$

Associativity for $i=j=1, k=2$ and for $i=1, j=k=2$ requires $\partial \zeta / \partial \bar{x}^{2}=0$ and $\partial \zeta / \partial \bar{x}^{1}=0$. Hence $\zeta(\bar{x})$ must be constant. The same arguments applied to $\tilde{x}^{a}, \tilde{x}^{b}$ and $\tilde{x}^{c}$ show that $\theta(\tilde{x})$ must 
also be constant. Therefore

rank 4: $\zeta(x)=$ const, $\quad \theta(x)=$ const.

This brings us back to the ordinary $\Theta$-constant Moyal 4-dimensional star product. We thus conclude that for rank 4, Rieffel's deformation framework does not provide generalizations to nonconstant star products in terms of associative algebras of functions. From our arguments it trivially follows that there is no 2-dimensional generalization in the sense of Rieffel of the Moyal product to nonconstant $\Theta(x)$, either.

\subsection{Case 2: $\operatorname{rank}(\Theta)=2$}

Constant rank 2 corresponds to one of the two functions $\zeta(x)$ and $\theta(x)$ being identically zero. So let us take $\zeta(x)=0$; if $\theta(x)=0$ one proceeds similarly. In this case integration over $\bar{y}$ and $\bar{z}$ in Eq. (2.4) is trivial and thus we are left with

$$
\left(f \star_{\theta} g\right)(x)=\frac{1}{(2 \pi)^{2}} \int d^{2} \tilde{u} d^{2} \tilde{v} f\left(\bar{x}, \tilde{x}-\frac{1}{2} \theta(x) S \tilde{u}\right) g(\bar{x}, \tilde{x}+\tilde{v}) e^{-i \tilde{u} \tilde{v}} .
$$

Here we have changed the notation to $\star_{\theta}$ to make explicit that we are in the case of rank 2 . The same arguments as for the rank 4 case show that Eq. (2.8) realizes the commutation relations (2.5). The Jacobi identity can also be analyzed in the same manner as for rank 4 . The difference is that now it does not restrict the dependence of $\theta(x)$ on $x$. Associativity, however, does, for we now arrive at

$$
\left(\tilde{x}^{a} \star_{\theta} \tilde{x}^{b}\right) \star_{\theta} \tilde{x}^{c}-\tilde{x}^{a} \star_{\theta}\left(\tilde{x}^{b} \star_{\theta} \tilde{x}^{c}\right)=-\frac{1}{4} \theta(x)\left[\varepsilon^{a b} \varepsilon^{c d} \frac{\partial \theta}{\partial \tilde{x}^{d}}+\varepsilon^{b c} \varepsilon^{a d} \frac{\partial \theta}{\partial \tilde{x}^{d}}\right]
$$

and for the right-hand side to vanish for all $a, b, c=1,2$ we need $\partial \theta / \partial \tilde{x}^{1}=0$ and $\partial \theta / \partial \tilde{x}^{2}=0$. Hence $\theta(x)$ may only depend on $\bar{x}$,

$\operatorname{rank} 2: \quad \theta(x)=\theta(\bar{x})$.

Once we have this, we prove associativity for arbitrary functions $f, g$ and $h$ on $\mathbb{R}^{4}$. For the left-hand side in Eq. (2.6) we have

$$
\begin{aligned}
{\left[\left(f \star_{\theta} g\right) \star_{\theta} h\right](x)=} & \frac{1}{(2 \pi)^{4}} \int d^{2} \tilde{u}_{1} d^{2} \tilde{v}_{1} d^{2} \tilde{u}_{2} d^{2} \tilde{v}_{2} e^{-i\left(\tilde{u}_{1} \tilde{v}_{1}+\tilde{u}_{2} \tilde{v}_{2}\right)} \\
& \times f\left(\bar{x}, \tilde{x}-\frac{1}{2} \theta(\bar{x}) S\left(\tilde{u}_{1}+\tilde{u}_{2}\right)\right) \\
& \times g\left(\bar{x}, \tilde{x}-\frac{1}{2} \theta(\bar{x}) S \tilde{u}_{2}+\tilde{v}_{1}\right) h\left(\bar{x}, \tilde{x}+\tilde{v}_{2}\right) .
\end{aligned}
$$

Making the changes $\tilde{v}_{1} \rightarrow \tilde{v}_{1}+\frac{1}{2} \theta(\bar{x}) S \tilde{u}_{2}$ and $\tilde{u}_{2} \rightarrow \tilde{u}_{2}-\tilde{u}_{1}$, we obtain after a plane wave integration

$$
\frac{1}{(2 \pi)^{2}} \int d^{2} \tilde{u} d^{2} \tilde{v} f\left(\bar{x}, \tilde{x}-\frac{1}{2} \theta(\bar{x}) S \tilde{u}\right) g(\bar{x}, \tilde{x}+\tilde{v}) h\left(\bar{x}, \tilde{x}+\tilde{v}+\frac{1}{2} \theta(\bar{x}) S \tilde{u}\right) e^{-i \tilde{u} \tilde{v}} .
$$

For the other side we have 


$$
\begin{aligned}
{\left[f \star_{\theta}\left(g \star_{\theta} h\right)\right](x)=} & \frac{1}{(2 \pi)^{4}} \int d^{2} \tilde{u}_{1} d^{2} \tilde{v}_{1} d^{2} \tilde{u}_{2} d^{2} \tilde{v}_{2} e^{-i\left(\tilde{u}_{1} \tilde{v}_{1}+\tilde{u}_{2} \tilde{v}_{2}\right)} f\left(\bar{x}, \tilde{x}-\frac{1}{2} \theta(\bar{x}) S \tilde{u}_{2}\right) \\
& \times g\left(\bar{x}, \tilde{x}-\frac{1}{2} \theta(\bar{x}) S \tilde{u}_{1}+\tilde{v}_{2}\right) h\left(\bar{x}, \tilde{x}+\tilde{v}_{1}+\tilde{v}_{2}\right) .
\end{aligned}
$$

Performing the changes $\tilde{v}_{2} \rightarrow \tilde{v}_{2}-\tilde{v}_{1}$ and $\tilde{v}_{1} \rightarrow \tilde{v}_{1}-\frac{1}{2} \theta(\bar{x}) S \tilde{u}_{1}$ in this order, and integrating over $\tilde{u}_{1}$ and $\tilde{v}_{1}$, we obtain

$$
\frac{1}{(2 \pi)^{2}} \int d^{2} \tilde{u} d^{2} \tilde{v} f\left(\bar{x}, \tilde{x}-\frac{1}{2} \theta(\bar{x}) S \tilde{u}\right) g\left(\bar{x}, \tilde{x}+\tilde{v}-\frac{1}{2} \theta(\bar{x}) S \tilde{u}\right) h(\bar{x}, \tilde{x}+\tilde{v}) e^{-i \tilde{u} \tilde{v}} .
$$

Changing now $\tilde{v} \rightarrow \tilde{v}+\frac{1}{2} \theta(\bar{x}) S \tilde{u}$ we reproduce (2.9), which proves associativity.

Appendix A contains a discussion of other basic properties of this star product. Among them, it is convenient to mention here the trace property and the Leibniz rule. The trace property

$$
\int d^{2} \tilde{x} d^{2} \bar{x}\left(f \star_{\theta} g\right)(\bar{x}, \tilde{x})=\int d^{2} \tilde{x} d^{2} \bar{x} f(\bar{x}, \tilde{x}) g(\bar{x}, \tilde{x})
$$

follows from Eq. (2.8) and some simple changes of variables. As concerns the Leibniz rule, differentials with respect to $\tilde{x}$ coordinates satisfy it, for

$$
\frac{\partial}{\partial \tilde{x}^{a}}\left(f \star_{\theta} g\right)=\frac{\partial f}{\partial \tilde{x}^{a}} \star_{\theta} g+f \star_{\theta} \frac{\partial g}{\partial \tilde{x}^{a}},
$$

whereas derivatives with respect to $\bar{x}$ coordinates do not. Indeed, acting with $\partial / \partial \bar{x}_{j}$ on Eq. (2.8), recalling that $\theta$ only depends on $\bar{x}$ and integrating by parts, we have

$$
\frac{\partial}{\partial \bar{x}^{j}}\left(f \star_{\theta} g\right)=\frac{\partial f}{\partial \bar{x}^{j}} \star_{\theta} g+f \star_{\theta} \frac{\partial g}{\partial \bar{x}^{j}}+\frac{i}{2} \frac{\partial \theta}{\partial \bar{x}^{j}} \varepsilon^{a b}\left(\frac{\partial f}{\partial \tilde{x}^{a}} \star_{\theta} \frac{\partial g}{\partial \tilde{x}^{b}}\right) .
$$

As a consequence, the star product constructed here is not an isospectral deformation in the sense of Connes and Landi [33,34], since commutators of the ordinary Dirac operator on $\mathbb{R}^{4}$ with left or right star multiplication contain derivatives. In particular, the commutators are no longer bounded operators. The formulation of nonconstant noncommutativity spaces as Connes' spectral triples is likely to involve techniques of the noncommutative geometry of foliations [35].

The star product associated to $\theta(x)=\theta(\bar{x})$ and $\zeta(x)=0$ that we have just constructed can be viewed as follows. Suppose we take infinitely many copies of $\mathbb{R}^{2}$ and that on each copy we define an ordinary $\theta$-constant Moyal product, with $\theta$ being different for different copies. To account for this difference, we make $\theta$ to depend on two real arbitrary parameters $\bar{x}^{1}$ and $\bar{x}^{2}$, external to $\mathbb{R}^{2}$. Now we form the product of the deformed $\mathbb{R}^{2}$, which we denote $\mathbb{R}_{\theta(\bar{x})}^{2}$, with the range of the external parameters, which is nothing but $\mathbb{R}_{\bar{x}}^{2}$. This gives a foliation $\mathbb{R}_{\bar{x}}^{2} \rtimes \mathbb{R}_{\theta(\bar{x})}^{2}$ of $\mathbb{R}^{4}$, i.e. the structure we have. From this viewpoint, it is natural that this construction define a nonconstant $\Theta(x)$ star product. This is the only one that happens to be associative within the framework provided by Rieffel's deformation quantization method. The construction generalizes to other dimensions in different forms. Indeed, for $n \geqslant 3$ odd or even and $n-2 m \geqslant 1$, we can write $\mathbb{R}^{n} \simeq \mathbb{R}^{n-2 m} \rtimes \mathbb{R}^{2 m}$ and $x=\left(\bar{x}^{1}, \ldots, \bar{x}^{n-2 m}, \tilde{x}^{1}, \ldots, \tilde{x}^{2 m}\right)$. We can even substitute a manifold for $\mathbb{R}^{n-2 m}$. The arguments presented here all go through, thus ensuring associativity.

\subsection{A different rank 2 construction: foliation of $\mathbb{R}^{4}$ by radius-dependent generalized} Connes-Landi spheres

Thus far we have implicitly supposed that the transformations required to bring $\Theta(x)$ into the canonical form do not change the topology of $\mathbb{R}^{4}$, in regard to foliations. Star products induced 
by periodic actions within the Rieffel's recipe partially escape this situation. To illustrate this point, let us give an example of a nonconstant star product based on a different decomposition of $\mathbb{R}^{4}$, namely the foliation of $\mathbb{R}^{4}$ by radius-dependent generalized Connes-Landi spheres [33]. This construction also has rank 2 and, although lying outside the main line of this paper, we include it because of its novelty. On ordinary space $\mathbb{R}^{4}$ define double cylindrical coordinates $(R, \kappa, \rho, \phi)$ by

$$
\begin{aligned}
& x_{1}=R \cos \kappa \cos \phi, \quad x_{2}=R \cos \rho \sin \phi, \\
& x_{3}=R \sin \rho \sin \phi, \quad x_{4}=R \sin \kappa \cos \phi,
\end{aligned}
$$

where $0 \leqslant R<+\infty, 0 \leqslant \kappa, \rho<2 \pi$ and $0 \leqslant \phi \leqslant \pi / 2$. Then consider a foliation $\mathbb{R}^{+} \rtimes \mathbb{S}_{\omega}^{3}$ of $\mathbb{R}^{4}$ by spheres of the Connes-Landi type (a continuous field of unital algebras) with a variable noncommutativity parameter $\omega=\omega(R)$, a smooth and strictly positive function. Define finally the star product through

$$
\begin{aligned}
\left(f \star_{\omega} h\right)\left(R, e^{i \kappa}, e^{i \rho}, \phi\right)= & \frac{1}{(2 \pi)^{2}} \int d^{2} u d^{2} t e^{-i u t} f\left(R, e^{i \kappa-i \omega(R) u_{2} / 2}, e^{i \rho+i \omega(R) u_{1} / 2}, \phi\right) \\
& \times h\left(R, e^{i \kappa+i t_{1}}, e^{i \rho+i t_{2}}, \phi\right),
\end{aligned}
$$

where $u=\left(u_{1}, u_{2}\right)$ and $t=\left(t_{1}, t_{2}\right)$. If functions on $\mathbb{R}^{4}$ are isotypically decomposed,

$$
f=\sum_{\mathbf{r} \in \mathbb{Z}_{2}} e^{-i\left(r_{1} \kappa+r_{2} \rho\right)} f_{\mathbf{r}}(R, \phi)
$$

the star product takes the form

$$
\left(f \star_{\omega} h\right)(R, \kappa, \rho, \phi)=\sum_{\mathbf{r}, \mathbf{s} \in \mathbb{Z}_{2}} e^{-i\left(r_{1}+s_{1}\right) \kappa} e^{-i\left(r_{2}+s_{2}\right) \rho} e^{i \omega(R) \mathbf{s} S \mathbf{r}} f_{\mathbf{r}}(R, \phi) h_{\mathbf{s}}(R, \phi) .
$$

The same analytical properties as for the $\mathbb{R}^{2} \rtimes \mathbb{R}_{\theta}^{2}$ foliation discussed in Section 2.2 and Appendix A are easily seen to also hold for this product.

\section{Traveling noncommutative lumps}

The nonconstant product $\star_{\theta}$ defined in Section 2 makes possible to extend the construction by Gopakumar et al. [23] of noncommutative solitons to include propagating lumps. Let us recall in this regard that Ref. [23] considers the extrema problem for the functional

$$
V[\phi]=\int d^{2 N} x\left(\frac{1}{2} m^{2} \phi^{2}+\sum_{s=3}^{r} \frac{b_{s}}{s} \phi_{\star}^{s}\right),
$$

where $\phi_{\star}^{s}$ is a short-hand for the noncommutative $s$ th power

$$
\phi_{\star}^{s}=\phi \star \phi \star \cdots \star \phi
$$

constructed with the constant noncommutativity matrix

$$
\Theta=\left(\begin{array}{cccc}
\theta_{1} S & & & \\
& \theta_{2} S & & \\
& & \ddots & \\
& & & \theta_{N} S
\end{array}\right) .
$$


There it is shown that the corresponding field equation

$$
m^{2} \phi+\sum_{s=3}^{r} b_{s} \phi_{\star}^{s-1}=0
$$

has solutions of the form

$$
\phi_{\Theta}\left(\left(x_{1}, x_{2}\right), \ldots,\left(x_{N-1}, x_{N}\right)\right)=\prod_{i=1}^{N} \sum_{k_{i}=0}^{\infty} a_{k_{i}} f_{\theta_{i}, k_{i}}\left(x_{i}, x_{i+1}\right) .
$$

In this expression the coefficients $a_{k_{i}}$ take values in the set of solutions of the equation

$$
m^{2} z+\sum_{s=3}^{r} b_{s} z^{s-1}=0
$$

and the $f_{\theta_{i}, k_{i}}$ are given by

$$
f_{\theta_{i}, k_{i}}\left(x_{i}, x_{i+1}\right)=(-1)^{k_{i}} 2 e^{-\rho_{i}^{2} / \theta_{i}} L_{k_{i}}\left(\frac{2 \rho_{i}^{2}}{\theta_{i}}\right),
$$

with $\rho_{i}$ defined in terms of the Cartesian coordinates $\left(x_{1}, \ldots, x_{2 N}\right)$ as

$$
\rho_{\frac{i+1}{2}}^{2}=x_{i}^{2}+x_{i+1}^{2}, \quad i=1,3, \ldots, 2 N-1,
$$

and $L_{k}(x)$ denoting the ordinary Laguerre polynomial of order $k$. These solutions can be written in many different fashions, for it is enough $\left\{f_{\theta_{i}, k_{i}}\right\}$ to be a family of real orthogonal projectors of the two-dimensional Moyal algebra with parameter $\theta_{i}$. The choice in Eq. (3.4) corresponds to taking for such projectors the Wigner eigenfunctions of the harmonic oscillator [28-30]. It is also possible to construct radial solutions for $\theta_{1}=\cdots=\theta_{N}=\theta$ of the form

$$
\phi_{\theta}(\rho)=\sum_{M} a_{M}(-1)^{M} 2^{N} e^{-\rho^{2} / \theta} L_{M}^{N-1}\left(\frac{2 \rho^{2}}{\theta}\right), \quad M=0,1,2, \ldots,
$$

where $\rho$ is the radial coordinate in $\mathbb{R}^{2 N}$ and the coefficient $a_{M}$ takes values in the set of solutions of Eq. (3.3).

We now consider $(2 N+2)$-dimensional Minkowski space-time, with coordinates $\left(t, x, x_{1}\right.$, $\left.\ldots, x_{2 N}\right)$. From our analysis in Section 2 it is clear that $\phi_{\Theta}$ in Eq. (3.2) is still a solution in such a space-time of the field equation (3.1) when the noncommutative parameters $\theta_{i}$ become functions of $t$ and $x$. Furthermore, if each $\theta_{i}$ only depends on either the sum $t+x$ or the difference $t-x$, the field configuration $\phi_{\Theta}$ solves the equation

$$
\left(\partial_{t}^{2}-\partial_{x}^{2}\right) \phi+m^{2} \phi+\sum_{s=3}^{r} b_{s} \phi_{\star}^{s-1}=0 .
$$

Such solutions describe lumps traveling in the negative or positive directions of $x$ with velocity 1 . It is also obvious that $\phi_{\theta}(\rho)$ in (3.5) describes a spherical traveling lump for $\theta$ a function of $t \pm x$. However, the solutions to Eq. (3.6) discussed here are not solutions of the field equation that results from adding to the functional $V[\phi]$ the fully-fledged kinetic term $-\frac{1}{2}(\partial \phi)^{2}$. 


\section{Field theory for noncommutative $\lambda \phi^{4}$ with variable $\theta$. The effective action at one loop: generalities}

Let us next consider quantization of a classical field theory for the nonconstant $\theta(\bar{x})$ star product constructed in Section 2. Our starting point for this is a real field $\phi$ with classical action (free energy) in Euclidean space given by

$$
S[\phi]=\int d^{4} x\left[\frac{1}{2} \phi\left(\Delta+m^{2}\right) \phi+\frac{\lambda}{4 !} \phi \star \phi \star \phi \star \phi\right] .
$$

Here $\Delta$ is the Laplacian, $m^{2}$ is the mass squared of the field, $\lambda$ is the coupling constant and we have written $\star$ instead of $\star_{\theta}$ to lighten the notation. In our conventions the Laplacian is positive: $\Delta=-\partial^{\mu} \partial_{\mu}$. This action defines the classical theory. We assume the quantum theory to be defined by its generating path integral [36]

$$
Z[j]=\mathcal{N} \int[d \phi] \exp \left(-S[\phi]+\int d^{4} x j \phi\right),
$$

where $j(x)$ is the source with respect to which functional differentiation of $Z[j]$ generates Green functions, and $\mathcal{N}$ is a normalization factor such that $Z[0]=1$. The path integral for $Z$ [ $j]$ is to be understood as the formal perturbative series that results from expanding about the classical solutions $\varphi$ reducing to the trivial one when $j=0$ of the equation

$$
\left.\frac{\delta S[\phi]}{\delta \phi(x)}\right|_{\phi=\varphi}=j(x) .
$$

We may suppose $\varphi$ is of Schwartz class. Now, shifting in the vicinity of a solution $\varphi$ the integration variable $\phi \rightarrow \varphi+\phi$ and substituting in $Z[j]$, we have

$$
Z[j]=\mathcal{N} e^{-I_{0}[\varphi, j]} \int[d \phi] e^{-\left(I_{2}[\varphi, \phi]+O\left[\phi^{3}\right]\right)},
$$

where

$$
\begin{aligned}
& I_{0}[\varphi, j]=S[\varphi]-\int d^{4} x j \varphi, \\
& I_{2}[\varphi, \phi]=\int d^{4} x\left[\frac{1}{2} \phi\left(\Delta+m^{2}\right) \phi+\frac{\lambda}{12}(2 \varphi \star \varphi \star \phi \star \phi+\varphi \star \phi \star \varphi \star \phi)\right] .
\end{aligned}
$$

At first order in perturbation theory only the quadratic part $I_{2}$ in the exponential contributes to the path integral.

In what follows we will use the notation

$$
\begin{aligned}
& H_{0}=\Delta+m^{2}, \quad H=H_{0}+M, \\
& M=\frac{\lambda}{6}\left(L_{\varphi \star \varphi}+R_{\varphi \star \varphi}+R_{\varphi} L_{\varphi}\right),
\end{aligned}
$$

with $L_{f}$ and $R_{f}$ denoting left and right star multiplication by $f$. The operators $L_{f}$ and $R_{f}$ commute with each other. Furthermore, because $\varphi$ is real, and thanks to the trace property, $L_{\varphi}$ and $R_{\varphi}$ are selfadjoint. Hence

$$
L_{\varphi \star \varphi}+R_{\varphi \star \varphi}+R_{\varphi} L_{\varphi}=\frac{1}{2}\left(L_{\varphi}+R_{\varphi}\right)^{*}\left(L_{\varphi}+R_{\varphi}\right)+\frac{1}{2}\left(L_{\varphi}^{*} L_{\varphi}+R_{\varphi}^{*} R_{\varphi}\right),
$$


which shows strict positivity of $H$. Integrating then over $[d \phi]$ we obtain for the one-loop correction to the effective action ${ }^{2}$

$$
\Gamma_{1}[\varphi]=-\frac{1}{2} \int_{0}^{\infty} \frac{d t}{t} \operatorname{Tr}\left(e^{-t H}-e^{-t H_{0}}\right)
$$

where the exponential $e^{-t H_{0}}$ comes from the normalization factor $\mathcal{N}$. We want to study the UV divergences of $\Gamma_{1}[\varphi]$ and see whether they can be subtracted by local counterterms. It is well known that UV singularities arise from the small $t$ expansion of the integrand in Eq. (4.2). To regulate this expansion we therefore introduce a zeta function-like regularization and consider instead

$$
\Gamma_{1}[\varphi]=-\frac{1}{2} \int_{0}^{\infty} \frac{\mu^{2 \epsilon} d t}{t^{1-\epsilon}} \operatorname{Tr}\left(e^{-t H}-e^{-t H_{0}}\right) .
$$

We must find a suitable method to compute the singularities that occur at $\epsilon \rightarrow 0$ when expanding in powers of $t$ the integrand in this expression. Because of the presence of the 'background field' $\theta(\bar{x})$ we work in configuration space.

In position space two methods have been proposed to compute the UV divergences for constant $\theta$. The first one, employed in Refs. [31,37], computes $e^{-t H}$ using Campbell-BakerHausdorff $(\mathrm{CBH})$ or Zassenhaus-type formulae. However, this does not work well for nonconstant $\theta(\bar{x})$. Instead we use the method that in the physics literature goes under the name of covariant perturbation theory [24], whereas to mathematicians is known as the Duhamel or "expansional" series [38]. For the sake of completeness, we sketch a two-line derivation for it. Since the standard power series for the exponential $e^{-t\left(H_{0}+M\right)}$ is of little use as $H_{0}$ and $M$ do not commute, it is better to think of $e^{-t\left(H_{0}+M\right)}$ as the solution of the differential equation

$$
\frac{d Y(t)}{d t}=-Y(t)\left(H_{0}+M\right)
$$

with initial condition $Y(0)=1$. Writing $Y(t)=X(t) e^{-t H_{0}}$, we obtain for $X$ the new differential equation

$$
\frac{d X(t)}{d t}=-X(t) M(t)
$$

with $M(t)=e^{-t H_{0}} M e^{t H_{0}}$. Its iterative solution reads

$$
e^{-t H}=\sum_{n=0}^{\infty} K_{n}(t)
$$

where $K_{0}(t)=e^{-t H_{0}}$ and

$$
K_{n}(t)=(-)^{n} \int_{0}^{t} d \sigma_{n} \int_{0}^{\sigma_{n}} d \sigma_{n-1} \cdots \int_{0}^{\sigma_{2}} d \sigma_{1} e^{-\sigma_{1} H_{0}} M e^{-\left(\sigma_{2}-\sigma_{1}\right) H_{0}} \cdots M e^{-\left(t-\sigma_{n}\right) H_{0}}
$$

\footnotetext{
${ }^{2}$ Recalling from Section 3 that $L_{\varphi}$ and $R_{\varphi}$ for $\varphi$ of Schwartz class are bounded, so that $M$ is bounded, one can generalize the arguments in Ref. [31] to prove a priori that the difference $e^{-t H}-e^{-t H_{0}}$ is trace-class for all $t>0$.
} 
for $n \geqslant 1$. Simple changes of variables [24] yield for the traces of the operators $K_{n}(t)$

$$
\operatorname{Tr} K_{n}(t)=\frac{(-t)^{n}}{n} \int_{0}^{\infty} d \alpha_{1} \cdots d \alpha_{n} \delta\left(1-\sum \alpha_{i}\right) \operatorname{Tr}\left(M e^{-t \alpha_{1} H_{0}} M e^{-t \alpha_{2} H_{0}} \cdots M e^{-t \alpha_{n} H_{0}}\right),
$$

so that only $n-1$ integrals need be performed to compute $\operatorname{Tr} K_{n}(t)$. Furthermore, since $M$ is positive and bounded from above, it follows that

$$
\operatorname{Tr} K_{n}(t) \sim t^{n-2} \quad \text { as } t \downarrow 0 .
$$

Hence, only

$$
\operatorname{Tr} K_{1}=-t \operatorname{Tr} M e^{-t H_{0}}
$$

and

$$
\operatorname{Tr} K_{2}=\frac{1}{2} t^{2} \int_{0}^{1} d \sigma \operatorname{Tr}\left[M e^{-t \sigma H_{0}} M e^{-t(1-\sigma) H_{0}}\right]
$$

will contribute to the divergent part of the regularized effective action as $\epsilon \rightarrow 0$. These two contributions correspond precisely to the two and four-point 1PI Green functions.

Because the procedure seems not as well known as perhaps it should be, we summarize the advantages of the covariant perturbation method:

- CBH-like series only converge for small $t$, whereas the convergence properties of the Duhamel perturbation expansion are so good that it not only adds up when $M$ is a bounded perturbation of an unbounded $A$ (the case here), but also for unbounded perturbations under appropriate circumstances.

- Small $t$ expansions by definition contain no information about $t \uparrow \infty$. In fact, to study $t \uparrow \infty$, one should regard Schwinger-DeWitt formulae as asymptotic expansions in the inverse mass squared, as it is the $e^{-t m^{2}}$ dampening factor that protects the integral (4.3) from infrared problems. The question has been tackled in the context of covariant perturbation theory [24] for ordinary quantum field theory and it has been proved that in four dimensions, irrespectively of mass, each term $\operatorname{Tr} K_{n}$ (for $n \geqslant 1$ ) of the expansion goes as $1 / t$ like $t \uparrow \infty$. Then the effective action always exists. To our knowledge, this question is open for NCFT.

- The Duhamel series has a cohomological meaning. Each of its terms is a cocycle in the entire cyclic cohomology of the Connes bicomplex, namely the Jaffe-Lesniewski-Osterwalder cocycle [5,39].

- Because the Duhamel expansion is essentially an expansion in powers of $M$, and $M$ is of order two in $\varphi$, each term gives directly a $2 n$-point 1 PI function. From this point of view, the $\alpha_{i}$-parameters are essentially Schwinger parameters for the propagators.

There is a price to pay for all these benefits, and that is nonlocality, making it more difficult to extract the singularities at $\epsilon \rightarrow 0$. We are bound to meet here two different types of nonlocality. The first one concerns the nonlocality of the Duhamel development itself. This comes from the entanglement of the heat kernel semigroups with the effective potentials. In the NCFT case with constant noncommutativity, it is well known that there are nonlocal contributions from both the planar and nonplanar sectors of the theory. We expect nonlocalities of this type to also occur here, may be worsened now by variability of $\theta(\bar{x})$. 


\section{The two-point sector of the effective action}

We have already mentioned in the last section that only $K_{1}(t)$ and $K_{2}(t)$ generate singularities at $\epsilon \rightarrow 0$, so that for the regularized effective action (4.3) we can write

$$
\Gamma_{1}[\varphi]=\Gamma_{1}^{(2)}[\varphi]+\Gamma_{1}^{(4)}[\varphi]+O(\epsilon)
$$

where

$$
\Gamma_{1}^{(2 n)}[\varphi]=-\frac{1}{2} \int_{0}^{\infty} \frac{\mu^{2 \epsilon} d t}{t^{1-\epsilon}} \operatorname{Tr} K_{n}(t), \quad n=1,2 .
$$

We compute $\Gamma_{1}^{\epsilon,(2)}[\varphi]$ in this section and postpone $\Gamma_{1}^{\epsilon,(4)}[\varphi]$ to Section 6 . Using for $\operatorname{Tr} K_{1}(t)$ the expression in (4.6), we have

$$
\Gamma_{1}^{(2)}[\varphi]=\frac{\lambda \mu^{2 \epsilon}}{12} \int_{0}^{\infty} d t t^{\epsilon} e^{-t m^{2}} \operatorname{Tr}\left[\left(L_{\varphi \star \varphi}+R_{\varphi \star \varphi}+R_{\varphi} L_{\varphi}\right) e^{-t \Delta}\right] .
$$

We first calculate the traces of the three terms in the integrand and then integrate over $d t$. The $L_{\varphi \star \varphi}$ and $R_{\varphi \star \varphi}$ terms in Eq. (5.1) yield the same contribution to the trace, since for any function $f$ one has

$$
\operatorname{Tr}\left(R_{f} e^{-t \Delta}\right)=\operatorname{Tr}\left(L_{f} e^{-t \Delta}\right)=\operatorname{Tr}\left(M_{f} e^{-t \Delta}\right)=\frac{1}{(4 \pi t)^{2}} \int d^{4} x f(\bar{x}, \tilde{x}),
$$

where $M_{f}$ denotes the operator of ordinary multiplication by $f$. To prove this, we note that from the definition of star product in Eq. (2.8) it follows, in obvious notation, that

$$
\begin{aligned}
& \left\langle x\left|L_{f}\right| y\right\rangle=\frac{1}{(2 \pi)^{2}} \int d^{2} \tilde{u} d^{2} \tilde{v} e^{-i \tilde{u} \tilde{v}} f\left(\bar{x}, \tilde{x}-\frac{1}{2} \theta(\bar{x}) S \tilde{u}\right) \delta^{(2)}(\bar{x}-\bar{y}) \delta^{(2)}(\tilde{x}+\tilde{v}-\tilde{y}), \\
& \left\langle x\left|R_{f}\right| y\right\rangle=\frac{1}{(2 \pi)^{2}} \int d^{2} \tilde{u} d^{2} \tilde{v} e^{-i \tilde{u} \tilde{v}} f(\bar{x}, \tilde{x}+\tilde{v}) \delta^{(2)}(\bar{x}-\bar{y}) \delta^{(2)}\left(\tilde{x}-\frac{1}{2} \theta(\bar{x}) S \tilde{u}-\tilde{y}\right) .
\end{aligned}
$$

Taking e.g. $L_{f}$ and noting

$$
\left\langle x\left|e^{-t \Delta}\right| y\right\rangle=\frac{1}{(4 \pi t)^{2}} e^{-|x-y|^{2} / 4 t},
$$

we write

$$
\begin{aligned}
\operatorname{Tr}\left(L_{f} e^{-t \Delta}\right) & =\int d^{4} x d^{4} y\left\langle x\left|L_{f}\right| y\right\rangle\left\langle y\left|e^{-t \Delta}\right| x\right\rangle \\
& =\frac{1}{(2 \pi)^{2}} \int d^{4} x \int d^{2} \tilde{u} d^{2} \tilde{v} e^{-i \tilde{u} \tilde{v}} f\left(\bar{x}, \tilde{x}-\frac{1}{2} \theta(\bar{x}) S \tilde{u}\right) \frac{e^{-\tilde{v}^{2} / 4 t}}{(4 \pi t)^{2}} .
\end{aligned}
$$

Making now the change $\tilde{x} \rightarrow \tilde{x}+\frac{1}{2} \theta(\bar{x}) S \tilde{u}$ and integrating over $\tilde{u}$ and $\tilde{v}$, we obtain

$$
\operatorname{Tr}\left(L_{f} e^{-t \Delta}\right)=\frac{1}{(4 \pi t)^{2}} \int d^{4} x f(\bar{x}, \tilde{x}) .
$$


The proof for $R_{f}$ goes along the same lines. Next we move on to the third term in Eq. (5.1). Using similar arguments, it is straightforward to show that

$$
\operatorname{Tr}\left(R_{f} L_{f} e^{-t \Delta}\right)=\frac{1}{(2 \pi)^{2}} \int \frac{d^{4} x}{\theta^{2}(\bar{x})} \int d^{2} \tilde{u} f(\bar{x}, \tilde{x}) f(\bar{x}, \tilde{x}+\tilde{u}) \frac{e^{-t \tilde{u}^{2} / \theta^{2}(\bar{x})}}{4 \pi t} .
$$

Substituting the results (5.2) and (5.5) in Eq. (5.1) and integrating over $t$ we have

$$
\begin{aligned}
\Gamma_{1}^{(2)}[\varphi]= & \frac{\lambda m^{2}}{96 \pi^{2}}\left(\frac{\mu^{2}}{m^{2}}\right)^{\epsilon} \Gamma(-1+\epsilon) \int d^{4} x \varphi^{2}(x) \\
& +\frac{\lambda}{192 \pi^{3}}\left(\frac{\mu^{2}}{m^{2}}\right)^{\epsilon} \Gamma(\epsilon) \int \frac{d^{4} x}{\theta^{2}(\bar{x})} \int d^{2} \tilde{u} \varphi(\bar{x}, \tilde{x}) \varphi(\bar{x}, \tilde{x}+\tilde{u})\left[1+\frac{\tilde{u}^{2}}{m^{2} \theta^{2}(\bar{x})}\right]^{-\epsilon} .
\end{aligned}
$$

The first term gives a local contribution. Hence the divergence that occurs in it when $\epsilon \rightarrow 0$ can be subtracted by a local counterterm. By contrast, the second term in (5.6) is nonlocal and, as a result, the singularity that it develops as $\epsilon \rightarrow 0$ cannot be subtracted by a local counterterm, thus spoiling renormalizability. Although this is not the by now traditional UV/IR mixing of NCFT, it reveals a connection between the UV and IR sectors, since the UV singularity at $\epsilon \rightarrow 0$ in $\Gamma(\epsilon)$ gets mixed with the long distance correlation contained in the integral over $\tilde{u}$. Note also that in Eq. (5.6) we observe the traditional splitting of $\theta$-constant NCFT in a planar part (first term) with the same dependence on $\theta(\bar{x})$ as in the classical action, and a nonplanar part (second term) with a more complicated dependence.

Except for the fact that now $\theta(\bar{x})$ depends on $\bar{x}$, this nonlocality of UV divergences also occurs for $\theta$-constant NCFT. Just take $\theta(\bar{x})=\theta$ constant and the result of Ref. [7] is recovered. There it is also explained how the corresponding result in momentum space-see e.g. [40]—conceals the singularity of the $\epsilon \rightarrow 0$ limit. For constant $\theta$ one may think of avoiding the problem by considering noncommutativity matrices of rank $4[9,10]$ or by supersymmetrizing the theory $[12,14]$. In the nonconstant $\Theta(x)$ case, as seen in Section 3, associativity excludes rank 4 noncommutativity matrices. Supersymmetry remains unexplored.

When several vertices come into play, in the trace computations one has to deal with (sums and differences of) the noncommutativity function $\theta(\bar{x})$ evaluated at different points. This will mix further the UV and IR sectors of the theory. To better understand this mixing, and because we do not yet want to exclude nonlocal UV renormalizations in a theory with a nonlocal $x$-dependent background $\theta(\bar{x})$, which most likely is to be understood as an effective theory, we forge ahead, and undertake the calculation of the four-point part of the effective action. This will also allow us to discuss methods to deal with the apparent nonlocality of the Duhamel expansion.

\section{The four-point sector of the effective action}

In what follows we study $\Gamma_{1}^{(4)}$. Using (4.7) we have

$$
\Gamma_{1}^{(4)}=-\frac{\mu^{2 \epsilon}}{4} \int_{0}^{\infty} d t t^{1+\epsilon} \int_{0}^{1} d \sigma \operatorname{Tr}\left[M e^{-t \sigma H_{0}} M e^{-t(1-\sigma) H_{0}}\right] .
$$

Recalling the expression of $M$ in terms of left and right multiplication operators, using the invariance of the integral under $\sigma \rightarrow 1-\sigma$, cyclicity of the trace and the property

$$
\left\langle x\left|R_{f}\right| y\right\rangle=\left\langle x\left|L_{f^{*}}\right| y\right\rangle^{*}=\left\langle y\left|L_{f}\right| x\right\rangle,
$$


we obtain for $\Gamma_{1}^{(4)}$ the sum of four terms

$$
\Gamma_{X_{I}}^{(4)}=-\frac{\lambda^{2} \mu^{2 \epsilon}}{144} \int_{0}^{\infty} d t t^{1+\epsilon} \int_{0}^{1} d \sigma X_{I}(t, \sigma), \quad I=1, \ldots, 4
$$

with

$$
\begin{aligned}
& X_{1}=2 \operatorname{Tr}\left[L_{\varphi \star \varphi} e^{-t \sigma H_{0}} L_{\varphi \star \varphi} e^{-t(1-\sigma) H_{0}}\right], \\
& X_{2}=2 \operatorname{Tr}\left[L_{\varphi \star \varphi} e^{-t \sigma H_{0}} R_{\varphi \star \varphi} e^{-t(1-\sigma) H_{0}}\right], \\
& X_{3}=4 \operatorname{Tr}\left[L_{\varphi \star \varphi} e^{-t \sigma H_{0}} R_{\varphi} L_{\varphi} e^{-t(1-\sigma) H_{0}}\right], \\
& X_{4}=\operatorname{Tr}\left[R_{\varphi} L_{\varphi} e^{-t \sigma H_{0}} R_{\varphi} L_{\varphi} e^{-t(1-\sigma) H_{0}}\right] .
\end{aligned}
$$

We shall see below that $\Gamma_{X_{1}}^{(4)}$ becomes singular at $\epsilon \rightarrow 0$, whereas $\Gamma_{X_{I}}^{(4)}$ is finite for $I=2,3,4$.

\subsection{UV divergences}

Let us first consider $\Gamma_{X_{1}}^{(4)}$. Using the expressions for the matrix elements of $L_{f}$ and $R_{f}$ in Eqs. (5.3) and (5.4), it is straightforward to see that $X_{1}$ can be recast as

$$
\begin{aligned}
X_{1}= & \frac{1}{(2 \pi)^{4}} \int d^{4} x d^{4} z \int d^{2} \tilde{u} d^{2} \tilde{v} d^{2} \tilde{u}^{\prime} d^{2} \tilde{v}^{\prime} e^{-i\left(\tilde{u} \tilde{v}+\tilde{u}^{\prime} \tilde{v}\right)}(\varphi \star \varphi)\left(x-\frac{1}{2} \theta(\bar{x}) S \tilde{u}\right) \\
& \times(\varphi \star \varphi)\left(z-\frac{1}{2} \theta(\bar{z}) S \tilde{u}^{\prime}\right)\left\langle x+\tilde{v}\left|e^{-t \sigma H_{0}}\right| z\right\rangle\left\langle z+\tilde{v}^{\prime}\left|e^{-t(1-\sigma) H_{0}}\right| x\right\rangle .
\end{aligned}
$$

We now make the changes

$$
\tilde{x} \rightarrow \tilde{x}+\frac{1}{2} \theta(\bar{x}) S \tilde{u}, \quad \tilde{v} \rightarrow \tilde{v}-\frac{1}{2} \theta(\bar{x}) S \tilde{u}, \quad \tilde{v}^{\prime} \rightarrow \tilde{v}^{\prime}+\frac{1}{2} \theta(\bar{x}) S \tilde{u},
$$

integrate over $\tilde{u}$ and $\tilde{v}$ and in the result perform

$$
\tilde{z} \rightarrow \tilde{z}+\frac{1}{2} \theta(\bar{z}) S \tilde{u}^{\prime}, \quad \tilde{v}^{\prime} \rightarrow \tilde{v}^{\prime}-\frac{1}{2} \theta(\bar{z}) S \tilde{u}^{\prime} .
$$

Proceeding in this way we obtain

$$
\begin{aligned}
X_{1}= & \frac{1}{(2 \pi)^{2}} \int d^{4} x d^{4} z \int d^{2} \tilde{u} d^{2} \tilde{v} e^{-i \tilde{u} \tilde{v}}(\varphi \star \varphi)(x)(\varphi \star \varphi)(z) \\
& \times\left\langle x+\frac{1}{2}[\theta(\bar{x})-\theta(\bar{z})] S \tilde{u}\left|e^{-t \sigma H_{0}}\right| z\right\rangle\left\langle z+\tilde{v}\left|e^{-t(1-\sigma) H_{0}}\right| x\right\rangle .
\end{aligned}
$$

Doing now the Gaussian integrations over $\tilde{u}$ and $\tilde{v}$ and substituting in the expression for $\Gamma_{X_{1}}^{(4)}$, we arrive at

$$
\Gamma_{X_{1}}^{(4)}=-\frac{\lambda^{2} \mu^{2 \epsilon}}{72(2 \pi)^{4}} \int d^{4} x d^{4} z \int_{0}^{\infty} d t t^{-1+\epsilon} e^{-t m^{2}} \int_{0}^{1} d \sigma(\varphi \star \varphi)(x)(\varphi \star \varphi)(z) K(x, z ; t, \sigma),
$$

where the kernel $K(x, z ; t, \sigma)$ is given by 


$$
\begin{aligned}
K(x, z ; t, \sigma)= & \frac{1}{4 t \sigma(1-\sigma)} \exp \left[-\frac{(\bar{x}-\bar{z})^{2}}{4 t \sigma(1-\sigma)}\right] \frac{1}{4 t \sigma(1-\sigma)+\theta_{-}^{2}(\bar{x}, \bar{z}) / t} \\
& \times \exp \left[-\frac{(\tilde{x}-\tilde{z})^{2}}{4 t \sigma(1-\sigma)+\theta_{-}^{2}(\bar{x}, \bar{z}) / t}\right]
\end{aligned}
$$

and by definition

$$
\theta_{ \pm}(\bar{x}, \bar{z})=\frac{1}{2}[\theta(\bar{x}) \pm \theta(\bar{z})]
$$

To analyze this expression we consider first the case in which $\theta_{-}=0$. Eq. (6.3) then takes the form

$$
\begin{aligned}
\Gamma_{X_{1}, \theta_{-}=0}^{(4)}= & -\frac{\lambda^{2} \mu^{2 \epsilon}}{72(4 \pi)^{2}} \int d^{4} x d^{4} z(\varphi \star \varphi)(x) \int_{0}^{\infty} d t \frac{e^{-t m^{2}}}{t^{1-\epsilon}} \\
& \times \int_{0}^{1} d \sigma \frac{e^{-|x-z|^{2} / 4 t \sigma(1-\sigma)}}{[4 \pi t \sigma(1-\sigma)]^{2}}(\varphi \star \varphi)(z) .
\end{aligned}
$$

This integral can be written as

$$
\Gamma_{X_{1}, \theta_{-}=0}^{(4)}=-\frac{\lambda^{2} \mu^{2 \epsilon}}{72(4 \pi)^{2}} \int d^{4} x \int_{0}^{\infty} d t \frac{e^{-t m^{2}}}{t^{1-\epsilon}} \int_{0}^{1} d \sigma(\varphi \star \varphi)(x)\left[e^{-t \sigma(1-\sigma) \Delta}(\varphi \star \varphi)\right](x) .
$$

Integrating over $t$, expanding in powers of $\epsilon$ and integrating over $\sigma$, we obtain

$$
\Gamma_{X_{1}, \theta_{-}=0}^{(4)}=-\frac{\lambda^{2}}{72(4 \pi)^{2}} \int d^{4} x(\varphi \star \varphi)(x)\left[\frac{1}{\epsilon}-\gamma-\ln \left(\frac{m^{2}+\Delta / 6}{\mu^{2}}\right)+O(\epsilon)\right](\varphi \star \varphi)(x) .
$$

This gives a local UV divergence, which in our regularization scheme is characterized by a pole in $\epsilon$. It is worth mentioning the nonlocal aspect of (6.3). Note that even if $\theta=0$, which corresponds to the ordinary commutative $\lambda \phi^{4}$ model, formula (6.5) looks nonlocal. The reason for that is the intrinsic nonlocality of the Duhamel method. This is the apparent nonlocality to which we referred at the end of Section 3.

If $\theta_{-} \neq 0$, we divide the domain of integration for $t$ into two parts: from 0 to $\alpha$ and from $\alpha$ to $\infty$, with $\alpha>0$. The contribution from $\alpha$ to $\infty$ is finite for $\epsilon \rightarrow 0$. That from 0 to $\alpha$ becomes singular at $\epsilon \rightarrow 0$. To find the singularity we recall the distributional identity

$$
\lim _{\alpha \downarrow 0} \frac{1}{(\pi \alpha)^{k / 2}} e^{-x^{2} / \alpha}=\delta^{k}(x) \quad \text { for } x \in \mathbb{R}^{k},
$$

where $\delta^{k}$ denotes the $k$-dimensional delta function. This gives for the kernel $K(x, z ; t, \sigma)$ the following behavior for small $t$ :

$$
\begin{aligned}
\lim _{t \downarrow 0} K(x, z ; t, \sigma) & =\lim _{t \downarrow 0} \frac{\delta^{2}(\bar{x}-\bar{z})}{4 t \sigma(1-\sigma)+\theta_{-}^{2}(\bar{x}, \bar{z}) / t} \exp \left[-\frac{(\tilde{x}-\tilde{z})^{2}}{4 t \sigma(1-\sigma)+\theta_{-}^{2}(\bar{x}, \bar{z}) / t}\right] \\
& =\delta^{4}(x-z) .
\end{aligned}
$$

Taking into consideration the factor $t^{-1+\epsilon} e^{-t m^{2}}$, we obtain for the singular part of $\Gamma_{X_{1}}^{(4)}$, after integration over $t$ and $\sigma$, 


$$
\Gamma_{X_{1}, \text { sing }}^{(4)}=-\frac{\lambda^{2}}{72(4 \pi)^{2} \epsilon} \int d^{4} x[(\varphi \star \varphi)(\varphi \star \varphi)](x) .
$$

We thus recover the same singularity (pole part) as for the $\theta_{-}=0$ case, which can be subtracted by adding a local counterterm to the classical action. Note in this regard that $K(x, z ; t, \sigma)$ has the same behavior for small $t$ as that of the kernel

$$
\frac{e^{-|x-z|^{2} / 4 t \sigma(1-\sigma)}}{[4 \pi t \sigma(1-\sigma)]^{2}}
$$

corresponding to $\theta_{-}=0$. Let us, nevertheless, remark that in NCFT at constant $\theta$ the contribution of $X_{1}$ is purely planar, whereas for nonconstant $\theta$ it is not. In fact, in Eq. (6.3) the finite planar and nonplanar contributions to the effective action thoroughly mix. With this we complete the analysis of $\Gamma_{X_{1}}^{(4)}$ in Eq. (6.1). Let us now move to that of $\Gamma_{X_{I}}^{(4)}$ for $I=2,3,4$.

\subsection{Finite contributions}

Using the expressions for the matrix elements of $L_{f}$ and $R_{f}$, and proceeding similarly as for $X_{1}$, it is not difficult to obtain for $X_{2}$

$$
\begin{aligned}
X_{2}= & \frac{1}{(2 \pi)^{2}} \int d^{4} x d^{4} z \int d^{2} \tilde{u} d^{2} \tilde{v} e^{-i \tilde{u} \tilde{v}}(\varphi \star \varphi)(x)(\varphi \star \varphi)(z)\left\langle x+\theta_{+}(\bar{x}, \bar{z}) S \tilde{u}\left|e^{-t \sigma H_{0}}\right| z\right\rangle \\
& \times\left\langle z+\tilde{v}\left|e^{-t(1-\sigma) H_{0}}\right| x\right\rangle .
\end{aligned}
$$

This is the same expression as for $X_{1}$ in Eq. (6.2), with the only difference that instead of the half-difference $\theta_{-}(\bar{x}, \bar{z})$ we have the half-sum $\theta_{+}(\bar{x}, \bar{z})$. Slightly more complicated expressions involving $\theta_{+}(\bar{x}, \bar{z})$ can be obtained for $X_{3}$ and $X_{4}$. Since $\theta(\bar{x})$ is strictly positive, the half-sum will also be strictly positive and will act in Eq. (6.7) as a cut-off. This hints that the contribution from $X_{2}$ to the effective action has no singularities at $\epsilon=0$. In fact in Appendix B it is shown that $X_{2}, X_{3}$ and $X_{4}$ are bounded from above by a constant times $e^{-t m^{2}} / t$,

$$
\left|X_{I}\right| \leqslant C_{I} \frac{e^{-t m^{2}}}{t}, \quad I=2,3,4 \text {. }
$$

Then, upon integration over $t$ and $\sigma$, they give finite contributions to the effective action, since

$$
\left|\Gamma_{X_{I}}^{(4)}\right| \leqslant C_{I}^{\prime} \lambda^{2} \mu^{2 \epsilon} \int_{0}^{\infty} d t t^{\epsilon} e^{-t m^{2}}=C_{I}^{\prime} \lambda^{2}\left(\frac{\mu^{2}}{m^{2}}\right)^{\epsilon} \Gamma(\epsilon+1)=C_{I}^{\prime} \lambda^{2}+O(\epsilon) .
$$

All in all we conclude that the only divergence in the four-point part of the effective action is due to $X_{1}$, has the form (6.6) and, as already stated, can be subtracted by a local counterterm, posing no problem to renormalizability.

Note that having a nonconstant $\theta(\bar{x})$ does not solve the traditional UV/IR mixing. This is obvious from Eq. (6.7), for if we make $\theta$ approach zero, $\theta_{+}$also approaches zero and the divergences due to short distances $x \rightarrow z$ reappear.

\section{Conclusion}

In this paper we have considered the generalization within Rieffel's formalism of the Moyal product in $\mathbb{R}^{4}$ to noncommutative products with position-dependent noncommutativity parameters, and the formulation of quantum field theory for such generalized noncommutative products. 
In constructing such nonconstant noncommutative products, the requirement of associativity proves very restrictive and only allows a rank 2 matrix $\Theta(x)$ with entries $\Theta^{i j}(x)=0$ and $\Theta^{a b}(x)=\varepsilon^{a b} \theta(\bar{x})$. Here $x=\left(\bar{x}^{1}, \bar{x}^{2}, \tilde{x}^{1}, \tilde{x}^{2}\right)$ and $\theta(\bar{x})$ is an arbitrary positive sufficiently smooth bounded function. Perhaps more interesting is to regard this noncommutative product as a foliation $\mathbb{R}_{\bar{x}}^{2} \rtimes \mathbb{R}_{\theta(\bar{x})}^{2}$ of $\mathbb{R}^{4}$. This point of view makes possible to generalize this construction to $n \geqslant 3$ dimensions by writing $\mathbb{R}^{n} \simeq \mathbb{R}^{n-2 m} \rtimes \mathbb{R}^{2 m}$. The existence of these 'nonconstant' star products in dimension $n$, where noncommutativity is constrained to $2 m$ dimensions and depends on the other $n-2 m$ coordinates allows to generalize the existing noncommutative solitons in the literature [23] to traveling noncommutative lumps. In this regard, it would be interesting to investigate its usefulness in the search for other time-varying noncommutative classical solutions. It also suggests to consider them in connection with extra dimensions. Another interesting question concerns the interpretation of variable noncommutativity in relation with the quantum group approach to the Moyal product [41].

To do quantum field theory on our noncommutative $x$-dependent background, we have worked in position space and used the covariant perturbation method [24]. We have been able to perform explicit calculations at one loop for the $\lambda \phi^{4}$ model. Our computations for the two and four-point 1PI functions reveal that quantum field theory for nonconstant $\theta(\bar{x})$ essentially suffers from the same problems as for constant $\theta$. In this regard, the arbitrariness in the choice of $\theta(\bar{x})$ has not been of much use and the situation is essentially not better nor worse than for constant $\theta$.

We close by mentioning that the construction of star products with nonconstant $\Theta(x)$ in Minkowski space-time is more involved, as the orbits of $\Theta$ under the action of the Lorentz group have a more complicated structure than under orthogonal similarity. The construction found here provides an $x$-dependent generalization of a space-like or magnetic constant noncommutativity in Minkowski space-time, for which the effective action can be obtained by analytic continuation.

\section{Acknowledgements}

The authors thank J.C. Várilly for helpful comments. V.G. wishes to acknowledge the hospitality of the Department of Theoretical Physics of Universidad Complutense de Madrid, where this work was started. J.M.G.B. is very grateful to B. Booss-Bavnbek for making available to him unpublished notes on the Duhamel expansion. He also thanks MEC, Spain for support through a 'Ramón y Cajal' contract. F.R.R. is grateful to MEC, Spain for financial support through grant No. BFM2002-00950.

\section{Appendix A. Algebra property and boundedness of left and right multiplication}

We collect in this appendix other basic properties of the star product $\star_{\theta}$ defined in Section 2.2.

(1) Involutivity. From the definition given in Eq. (2.8) it is obvious that

$$
\left(f \star_{\theta} g\right)^{*}=g^{*} \star_{\theta} f^{*} .
$$

(2) Equivariance. For arbitrary $\tilde{u} \in \mathbb{R}^{2}$ and $M(x) \in S L(2, \mathbb{R})$, the following can be seen to hold:

$$
\begin{aligned}
& \left(f \star_{\theta} g\right)(\bar{x}, \tilde{x}+\tilde{u})=f(\bar{x}, \tilde{x}+\tilde{u}) \star_{\theta} g(\bar{x}, \tilde{x}+\tilde{u}), \\
& \left(f \star_{\theta} g\right)(\bar{x}, M(x) \tilde{x})=f(\bar{x}, M(x) \tilde{x}) \star_{\theta} g(\bar{x}, M(x) \tilde{x}) .
\end{aligned}
$$


(3) Algebra property. We have implicitly assumed that the functions $f, g, \ldots$ for which the star product $\star_{\theta}$ is defined and the function $\theta(\bar{x})$ satisfy good enough properties for the different objects that we have considered to be well defined. Let us now be more precise about this point. By $\mathcal{S}\left(\mathbb{R}^{4}\right)$ we denote the space of Schwartz (smooth, rapidly decreasing together with all derivatives) functions on $\mathbb{R}^{4}$. We recall that the standard Fréchet topology of $\mathcal{S}\left(\mathbb{R}^{4}\right)$ is given by the family of semi-norms

$$
p_{k, l}(f)=\sup _{|\alpha| \leqslant k,|\beta| \leqslant l} \sup _{x \in \mathbb{R}^{4}}\left|x^{\alpha} \partial^{\beta} f(x)\right|,
$$

or alternatively by

$$
q_{k, l}(f)=\sup _{|\alpha| \leqslant k,|\beta| \leqslant l} \sup _{\bar{x} \in \mathbb{R}^{2}}\left\{\int d^{2} \tilde{x}\left|x^{\alpha} \partial^{\beta} f(x)\right|\right\} .
$$

Since $\theta(\bar{x})>0$, we can rewrite the star product as

$$
\left(f \star_{\theta} g\right)(\bar{x}, \tilde{x})=\frac{1}{\pi^{2} \theta^{2}(\bar{x})} \int d^{2} \tilde{y} d^{2} \tilde{z} e^{-2 i(\tilde{x}-\tilde{y}) S(\tilde{x}-\tilde{z}) / \theta(\bar{x})} f(\bar{x}, \tilde{y}) g(\bar{x}, \tilde{z}) .
$$

Thus, if

$$
\frac{C_{1}}{\left(1+|\bar{x}|^{2}\right)^{k_{0}}} \leqslant \theta(\bar{x}) \leqslant C_{2},
$$

with $k_{0}$ a positive integer and $C_{1}, C_{2}>0$ constants, we get

$$
\begin{aligned}
p_{0,0}\left(f \star_{\theta} g\right) & \leqslant \frac{1}{\pi^{2}} \sup _{\bar{x} \in \mathbb{R}^{2}}\left\{\frac{1}{\theta(\bar{x})} \int d^{2} \tilde{y}|f(\bar{x}, \tilde{y})|\right\} \sup _{\bar{x} \in \mathbb{R}^{2}}\left\{\frac{1}{\theta(\bar{x})} \int d^{2} \tilde{z}|g(\bar{x}, \tilde{z})|\right\} \\
& \leqslant \frac{1}{C_{1}^{2} \pi^{2}}\left[\sum_{j=0}^{2 k_{0}}\left(\begin{array}{c}
2 k_{0} \\
j
\end{array}\right) q_{2 j, 0}(f)\right]\left[\sum_{i=0}^{2 k_{0}}\left(\begin{array}{c}
2 k_{0} \\
i
\end{array}\right) q_{2 i, 0}(g)\right] .
\end{aligned}
$$

For other values of $k$ and $l$, we obtain similar estimates, provided all the derivatives of the function $\theta(\bar{x})$ are polynomially bounded. Indeed, noting

$$
\begin{aligned}
\bar{x}^{i}\left(f \star_{\theta} g\right)(\bar{x}, \tilde{x}) & =\left(\bar{x}^{i} f\right) \star_{\theta} g(\bar{x}, \tilde{x})=f \star_{\theta}\left(\bar{x}^{i} g\right)(\bar{x}, \tilde{x}), \\
\tilde{x}^{a}\left(f \star_{\theta} g\right)(\bar{x}, \tilde{x}) & =f \star_{\theta}\left(\tilde{x}^{a} g\right)(\bar{x}, \tilde{x})+\frac{i}{2} \theta(\bar{x}) \varepsilon^{a b}\left(\frac{\partial f}{\partial \tilde{x}^{b}} \star_{\theta} g\right)(\bar{x}, \tilde{x}) \\
& =\left(\tilde{x}^{a} f\right) \star_{\theta} g(\bar{x}, \tilde{x})-\frac{i}{2} \theta(\bar{x}) \varepsilon^{a b}\left(f \star_{\theta} \frac{\partial g}{\partial \tilde{x}^{b}}\right)(\bar{x}, \tilde{x}),
\end{aligned}
$$

and using (2.10) and (2.11), we obtain by induction

$$
p_{k, l}\left(f \star_{\theta} g\right) \leqslant \sum_{i \in I, j \in J} K\left(C_{1}, k_{0}, k_{i}^{\prime}, k_{j}^{\prime}\right) q_{k_{j}^{\prime}, k_{j}}(f) q_{k_{i}^{\prime}, k_{i}}(g),
$$

for some finite sets $I, J$. This shows that the star product $\star_{\theta}$ is separately continuous, indeed jointly continuous, since $\mathcal{S}\left(\mathbb{R}^{4}\right)$ is a Fréchet space. Hence the space $\mathcal{S}\left(\mathbb{R}^{4}\right)$ endowed with the star product $\star_{\theta}$ is an associative and involutive Fréchet algebra with jointly continuous product. Under the same conditions the operators of left $L_{f}$ and right $R_{f}$ star multiplication by $f$ are bounded on the Hilbert space $L^{2}\left(\mathbb{R}^{4}\right)$. For $L_{f}$ this means that $\left\|L_{f} \psi\right\|=\left\|f \star_{\theta} \psi\right\|<\infty$ for all $f$ in $\mathcal{S}\left(\mathbb{R}^{4}\right)$ and all $\psi$ in $L^{2}\left(\mathbb{R}^{4}\right)$. To prove it, we make in Eq. (2.8) the change $\tilde{u} \rightarrow 2 \tilde{u} / \theta(\bar{x})$, use 
the identity

$$
e^{-2 i \tilde{u} \tilde{v} / \theta(\bar{x})}=\frac{1}{\left(1+|\tilde{u}|^{2}\right)^{k}}\left[1+\frac{1}{4} \theta^{2}(\bar{x}) \Delta_{\tilde{v}}\right]^{k} e^{-2 i \tilde{u} \tilde{v} / \theta(\bar{x})}
$$

for some integer $k>1$, and integrate by parts. For all $\psi \in L^{2}\left(\mathbb{R}^{4}\right)$ we then have, after further changing $\tilde{u} \rightarrow \tilde{x}-S^{-1} \tilde{u}$,

$$
\left|L_{f} \psi(\bar{x}, \tilde{x})\right| \leqslant \frac{1}{\pi^{2} \theta^{2}(\bar{x})} \int d^{2} \tilde{u} d^{2} \tilde{v}\left|\left[\left(1+\frac{1}{4} \theta^{2}(\bar{x}) \Delta_{\tilde{u}}\right)^{k} f\right](\bar{x}, \tilde{u})\right| \frac{\left|\left(U_{\tilde{v}} \psi\right)(\bar{x}, \tilde{x})\right|}{\left(1+|\tilde{v}|^{2}\right)^{k}},
$$

with $U_{\tilde{v}}$ the unitary operator of translation by $\tilde{v}$. Finally, we obtain

$$
\begin{aligned}
\left\|L_{f} \psi\right\|_{2} \leqslant & \frac{1}{\pi^{2}} \sup _{\bar{x} \in \mathbb{R}^{2}}\left\{\frac{1}{\theta^{2}(\bar{x})} \int d^{2} \tilde{u}\left|\left[\left(1+\frac{1}{4} \theta^{2}(\bar{x}) \Delta_{\tilde{u}}\right)^{k} f\right](\bar{x}, \tilde{u})\right|\right\}\|\psi\|_{2} \\
& \times \int d^{2} \tilde{v} \frac{1}{\left(1+|\tilde{v}|^{2}\right)^{k}},
\end{aligned}
$$

which is finite for $k>1$ since $f \in \mathcal{S}\left(\mathbb{R}^{4}\right)$ and $\theta(\bar{x})$ satisfies condition (A.1).

In our arguments in Section 2 we used coordinate functions not belonging to $\mathcal{S}\left(\mathbb{R}^{4}\right)$. That is a trivial rigor point, however, as they do belong to its multiplier algebra. The reader is referred in this regard to [30], as everything here works the same as in those references.

\section{Appendix B. Proof of Eq. (6.8)}

In this appendix we prove Eq. (6.8). We present a detailed discussion of the more involved case, namely $X_{4}$. The proofs for $X_{2}$ and $X_{3}$ go along the same lines and can be easily retrieved from the one presented here.

Using the expressions for the matrix elements of $L_{f}$ and $R_{f}$ in Eqs. (5.3) and (5.4), we obtain after some simple changes of variables and two plane wave integrations that

$$
\begin{aligned}
X_{4}= & \frac{1}{(2 \pi)^{4}} \int d^{4} x d^{4} z \int d^{2} \tilde{u} d^{2} \tilde{v} d^{2} \tilde{u}^{\prime} d^{2} \tilde{v}^{\prime} e^{-i\left(\tilde{u} \tilde{v}+\tilde{u}^{\prime} \tilde{v}^{\prime}\right)} \varphi(x+\tilde{v}) \varphi\left(x-\frac{1}{2} \theta(\bar{x}) S \tilde{u}-\tilde{v}\right) \\
& \times \varphi\left(z+\tilde{v}^{\prime}\right) \varphi\left(z-\frac{1}{2} \theta(\bar{z}) S \tilde{u}^{\prime}-\tilde{v}^{\prime}\right)\left\langle x-\frac{1}{2} \theta(\bar{x}) S \tilde{u}\left|e^{-t \sigma H_{0}}\right| z\right\rangle \\
& \times\left\langle z-\frac{1}{2} \theta(\bar{z}) S \tilde{u}^{\prime}\left|e^{-t(1-\sigma) H_{0}}\right| x\right\rangle .
\end{aligned}
$$

We now make the changes $\tilde{u} \rightarrow 2 \tilde{u} / \theta(\bar{x})$ and $\tilde{u}^{\prime} \rightarrow 2 \tilde{u}^{\prime} / \theta(\bar{z})$, use the identity (A.2) and integrate by parts, noting that the action of $\left[1+\frac{1}{4} \theta^{2}(\bar{x}) \Delta_{\tilde{v}}\right]^{k}$ on the product $\varphi(x+\tilde{v}) \varphi(x-S \tilde{u}-\tilde{v})$ generates a sum of the type

$$
\sum_{I+J=0, \ldots, 2 k}\left[P_{I}\left(\frac{1}{2} \theta(\bar{x}) \tilde{\partial}\right) \varphi\right](x+\tilde{v})\left[P_{J}\left(\frac{1}{2} \theta(\bar{x}) \tilde{\partial}\right) \varphi\right](x-S \tilde{u}-\tilde{v}),
$$

with $P_{I}(\cdot)$ and $P_{J}(\cdot)$ polynomials of order $I$ and $J$. This yields

$$
\left|X_{4}\right| \leqslant \frac{1}{\pi^{4}} \sum_{I+J=0, \ldots, 2 k} \int \frac{d^{4} x}{\theta^{2}(\bar{x})} \frac{d^{4} z}{\theta^{2}(\bar{z})} \frac{d^{2} \tilde{u} d^{2} \tilde{v}}{\left(1+|\tilde{u}|^{2}\right)^{k}} d^{2} \tilde{u}^{\prime} d^{2} \tilde{v}^{\prime}
$$




$$
\begin{aligned}
& \times\left|P_{I}\left(\frac{1}{2} \theta(\bar{x}) \tilde{\partial}\right) \varphi(x+\tilde{v})\right|\left|P_{J}\left(\frac{1}{2} \theta(\bar{x}) \tilde{\partial}\right) \varphi(x-S \tilde{u}-\tilde{v})\right| \\
& \times\left|\varphi\left(z+\tilde{v}^{\prime}\right)\right|\left|\varphi\left(z-S \tilde{u}^{\prime}-\tilde{v}^{\prime}\right)\right|\left\langle x-S \tilde{u}\left|e^{-t \sigma H_{0}}\right| z\right\rangle\left\langle z-S \tilde{u}^{\prime}\left|e^{-t(1-\sigma) H_{0}}\right| x\right\rangle .
\end{aligned}
$$

Making the change $\tilde{v} \rightarrow \tilde{v}-\tilde{x}$ and recalling that the norm $\|\cdot\|_{\infty}$ of a function $f$ defined on $\mathbb{R}^{n}$ is $\|f\|_{\infty}=\sup _{x \in \mathbb{R}^{n}}|f(x)|$, we have

$$
\begin{aligned}
\left|X_{4}\right| \leqslant & \frac{1}{\pi^{4}} K(\theta, k, \varphi) \int d^{4} x \frac{d^{4} z}{\theta^{2}(\bar{z})} \frac{d^{2} \tilde{u}}{\left(1+|\tilde{u}|^{2}\right)^{k}} d^{2} \tilde{u}^{\prime} d^{2} \tilde{v}^{\prime}\left|\varphi\left(z+\tilde{v}^{\prime}\right)\right| \\
& \times\left|\varphi\left(z-S \tilde{u}^{\prime}-\tilde{v}^{\prime}\right)\right|\left\langle x-S \tilde{u}\left|e^{-t \sigma H_{0}}\right| z\right\rangle\left\langle z-S \tilde{u}^{\prime}\left|e^{-t(1-\sigma) H_{0}}\right| x\right\rangle .
\end{aligned}
$$

The constant $K(\theta, k, \varphi)$ is given

$$
K(\theta, k, \varphi)=\sum_{I+J=0, \ldots, k} \sup _{\bar{x} \in \mathbb{R}^{2}}\left\{\frac{1}{\theta^{2}(\bar{x})}\left\|P_{J}\left(\frac{1}{2} \theta(\bar{x}) \tilde{\partial}\right) \varphi\right\|_{\infty} \int d^{2} \tilde{v}\left|P_{I}\left(\frac{1}{2} \theta(\bar{x}) \tilde{\partial}\right) \varphi(\bar{x}, \tilde{v})\right|\right\}
$$

and is a finite, since $\theta(\bar{x})$ is finite and nonrapidly decreasing by condition (A.1), and $\varphi$ is of Schwartz class.

The semigroup property

$$
\int d^{4} y\left\langle x\left|e^{-t_{1} H_{0}}\right| y\right\rangle\left\langle y\left|e^{-t_{2} H_{0}}\right| z\right\rangle=\left\langle x\left|e^{-\left(t_{1}+t_{2}\right) H_{0}}\right| z\right\rangle
$$

now allows us to perform the integral over $x$ in Eq. (B.1), with the result $\left\langle z-S \tilde{u}^{\prime}\left|e^{-t H_{0}}\right| z+S \tilde{u}\right\rangle$. Using this and making the changes $\tilde{z} \rightarrow \tilde{z}-\tilde{v}^{\prime}$ and $\tilde{v}^{\prime} \rightarrow \tilde{v}^{\prime}-\frac{1}{2} \tilde{u}^{\prime}$ we recast Eq. (B.1) as

$$
\left|X_{4}\right| \leqslant \frac{1}{4 \pi^{4}} K(\theta, k, \varphi) \int \frac{d^{4} z}{\theta^{2}(\bar{z})} \frac{d^{2} \tilde{u}}{\left(1+|\tilde{u}|^{2}\right)^{k}} d^{2} \tilde{u}^{\prime} d^{2} \tilde{v}^{\prime}|\varphi(z)|\left|\varphi\left(z-2 \tilde{v}^{\prime}\right)\right|\left\langle-S \tilde{u}^{\prime}\left|e^{-t H_{0}}\right| S \tilde{u}\right\rangle .
$$

Integration over $\tilde{u}$ and $\tilde{u}^{\prime}$ is now possible,

$$
\int \frac{d^{2} \tilde{u}}{\left(1+|\tilde{u}|^{2}\right)^{k}}=\frac{\pi}{k-1} \quad \text { and } \quad \int d^{2} \tilde{u}^{\prime}\left\langle-S \tilde{u}^{\prime}\left|e^{-t H_{0}}\right| S \tilde{u}\right\rangle=\frac{e^{-t m^{2}}}{4 \pi t} .
$$

As concerns integration over $z$ and $\tilde{v}^{\prime}$, we observe that, performing the change $\tilde{v}^{\prime} \rightarrow \frac{1}{2}\left(\tilde{z}-\tilde{v}^{\prime}\right)$,

$$
\int \frac{d^{4} z}{\theta(\bar{z})} d^{2} \tilde{v}^{\prime}|\varphi(z)|\left|\varphi\left(z-2 \tilde{v}^{\prime}\right)\right| \leqslant\|\varphi\|_{1} \sup _{\bar{z} \in \mathbb{R}^{2}}\left\{\frac{1}{4 \theta^{2}(\bar{z})} \int d^{2} \tilde{v}^{\prime}\left|\varphi\left(\bar{z}, \tilde{v}^{\prime}\right)\right|\right\} .
$$

All in all we have that $\left|X_{4}\right| \leqslant$ const $\times e^{-t m^{2}} / t$, as we wanted to prove.

\section{References}

[1] N. Seiberg, E. Witten, String theory and noncommutative geometry, JHEP 9909 (1999) 032.

[2] J.E. Moyal, Quantum mechanics as a statistical theory, Proc. Cambridge Philos. Soc. 45 (1949) 99.

[3] V. Gayral, J.M. Gracia-Bondía, B. Iochum, T. Schücker, J.C. Várilly, Moyal planes are spectral triples, Commun. Math. Phys. 264 (2004) 569.

[4] A. Connes, Gravity coupled with matter and the foundation of noncommutative geometry, Commun. Math. Phys. 182 (1996) 155.

[5] J.M. Gracia-Bondía, J.C. Várilly, H. Figueroa, Elements of Noncommutative Geometry, Birkhäuser, Boston, 2001.

[6] J. Gomis, T. Mehen, Space-time noncommutative field theories and unitarity, Nucl. Phys. B 591 (2000) 265. 
[7] V. Gayral, J.M. Gracia-Bondía, F. Ruiz Ruiz, Trouble with space-like noncommutative field theory, Phys. Lett. B 610 (2005) 141.

[8] D. Bahns, S. Doplicher, K. Fredenhagen, G. Piacitelli, Field theory on noncommutative spacetimes: Quasiplanar Wick products, Phys. Rev. D 71 (2005) 025022.

[9] D. Bahns, S. Doplicher, K. Fredenhagen, G. Piacitelli, On the unitary problem in space-time noncommutative theories, Phys. Lett. B 533 (2002) 178.

[10] K. Fujikawa, Path integral for space-time noncommutative field theory, Phys. Rev. D 70 (2004) 085006.

[11] K. Landsteiner, E. López, M.H.G. Tytgat, Excitations in hot noncommutative theories, JHEP 0009 (2000) 027; C.P. Martín, F. Ruiz Ruiz, Paramagnetic dominance, the sign of the beta function and UV/IR mixing in noncommutative $U(1)$, Nucl. Phys. B 597 (2001) 197.

[12] F. Ruiz Ruiz, Gauge-fixing independence of IR divergences in noncommutative $U(1)$, perturbative tachyonic instabilities and supersymmetry, Phys. Lett. B 502 (2001) 274.

[13] S. Minwalla, M.V. Raamsdonk, N. Seiberg, Noncommutative perturbative dynamics, JHEP 0002 (2000) 020.

[14] A. Matusis, L. Susskind, N. Toumbas, The IR/UV connection in the noncommutative gauge theories, JHEP 0012 (2000) 002.

[15] J.M. Gracia-Bondía, F. Lizzi, G. Marmo, P. Vitale, Infinitely many star-products to play with, JHEP 0204 (2002) 026.

[16] F. Lizzi, G. Mangano, G. Miele, M. Peloso, Cosmological perturbations and short distance physics from noncommutative geometry, JHEP 0206 (2002) 049.

[17] L. Dolan, C.R. Nappi, Noncommutativity in a time-dependent background, Phys. Lett. B 551 (2003) 369.

[18] X. Calmet, M. Wohlgennant, Effective field theories on noncommutative space-time, Phys. Rev. D 68 (2003) 025016.

[19] W. Behr, A. Sykora, Construction of gauge theories on curved noncommutative space-time, Nucl. Phys. B 698 (2004) 473.

[20] A. Hashimoto, K. Thomas, Dualities, twists and gauge theories with nonconstant noncommutativity, JHEP 0501 (2005) 033.

[21] L. Cornalba, R. Schiappa, Nonassociative star product deformations for D-brane worldvolumes in curved backgrounds, Commun. Math. Phys. 225 (2002) 33.

[22] M.A. Rieffel, Deformation Quantization for Actions of $\mathbb{R}^{d}$, Memoirs of the American Mathematical Society, vol. 506, American Mathematical Society, Providence, RI, 1993.

[23] R. Gopakumar, S. Minwalla, A. Strominger, Noncommutative solitons, JHEP 0005 (2000) 020.

[24] A.O. Barvinsky, G.A. Vilkovisky, Covariant perturbation theory (II). Second order in the curvature. General algorithms, Nucl. Phys. B 333 (1990) 471.

[25] P.B. Gilkey, Invariance Theory, the Heat Equation and the Atiyah-Singer Theorem, CRC Press, Boca Raton, 1995.

[26] M. Kontsevich, Deformation quantization of Poisson manifolds, Lett. Math. Phys. 66 (2003) 157.

[27] C.D. Fosco, G. Torroba, Noncommutative theories and general coordinate transformations, Phys. Rev. D 71 (2005) 065012.

[28] H.J. Groenewold, On the principles of elementary quantum mechanics, Physica 12 (1946) 405-460.

[29] M.S. Bartlett, J.E. Moyal, The exact transition probabilities of quantum-mechanical oscillators calculated by the phase-space method, Proc. Cambridge Philos. Soc. 45 (1949) 545-553.

[30] J.M. Gracia-Bondía, J.C. Várilly, Algebras of distributions suitable for phase-space quantum mechanics. I, J. Math. Phys. 29 (1988) 869;

J.C. Várilly, J.M. Gracia-Bondía, Algebras of distributions suitable for phase-space quantum mechanics. II. Topologies on the Moyal algebra, J. Math. Phys. 29 (1988) 880.

[31] V. Gayral, Heat-kernel approach to UV/IR mixing on isospectral deformation manifolds, hep-th/0412233, Ann. Inst. H. Poincaré, in press.

[32] V. Gayral, B. Iochum, J.C. Várilly, Dixmier traces on noncompact isospectral deformations, in preparation.

[33] A. Connes, G. Landi, Noncommutative manifolds, the instanton algebra and isospectral deformations, Commun. Math. Phys. 221 (2001) 141.

[34] A. Connes, M. Dubois-Violette, Noncommutative finite-dimensional manifolds. I. Spherical manifolds and related examples, Commun. Math. Phys. 230 (2002) 539.

[35] A. Connes, H. Moscovici, Hopf algebras, cyclic cohomology and the transverse index theorem, Commun. Math. Phys. 198 (1998) 198.

[36] C. Itzykson, J.B. Zuber, Quantum Field Theory, McGraw-Hill, New York, 1980.

[37] V. Gayral, B. Iochum, The spectral action for Moyal planes, hep-th/0402147, J. Math. Phys., in press.

[38] H. Araki, Expansional in Banach algebras, Ann. Sci. École Norm. Sup. 6 (1973) 67. 
[39] A. Connes, Noncommutative Geometry, Academic Press, San Diego, 1994.

[40] F. Ruiz Ruiz, UV/IR mixing and the Goldstone theorem in noncommutative field theory, Nucl. Phys. B 637 (2002) 143.

[41] R. Oeckl, Untwisting noncommutative $\mathbb{R}^{d}$ and the equivalence of quantum field theories, Nucl. Phys. B 581 (2000) 559 ;

M. Chaichian, P.P. Kulish, K. Nishijima, A. Tureanu, On a Lorentz-invariant interpretation of noncommutative space-time and its implications on noncommutative quantum field theory, Phys. Lett. B 604 (2004) 98. 